\title{
A Novel High Water-Resistant Aqueous Acrylate Emulsion, Part 1: Preparation and Characterization
}

\author{
B.K. Ren \\ School of Biological and Chemical Engineering \\ Zhejiang University of Science and Technology \\ College of Chemical Engineering \\ Zhejiang University of Technology \\ China \\ G.H. Li \\ College of Chemical Engineering \\ Zhejiang University of Technology \\ China
}

\author{
K.T. Hu \\ School of Biological and Chemical Engineering \\ Zhejiang University of Science and Technology \\ China
}

A.B. Wu, J.J. Li

Hangzhou Special Paper Industry Co. Ltd, China

\begin{abstract}
The acrylate copolymer emulsion with core-shell structure was successfully prepared via pre-emulsified semi-continuous seeded polymerization using SDS and OP-10 as compound emulsifier, and methyl methylacrylate (MMA), butyl acrylate (BA) and dodecafluoroheptyl methacrylate (DFMA) as main monomers. SEM and OCA were used to characterize the structure and the product, meanwhile, the influence of core-shell monomer ratio, the dosage of emulsifier, initiator and fluoride monomers on the reacting product properties were studied. Obvious ball structure could be seen clearly by the scanning electron microscopes when the hard monomer MMA matched with a moderate amount of DFMA as the shell in an optimized reaction condition. Detected with the help of particulate size description analyser, the latex particle which had uniformly size was found mono-dispersed.
\end{abstract}

Keywords-fluorinated acrylate; aqueous emulsion; core-shell structure; water-resistance; uniform particle size

\section{INTRODUCTION}

Aqueous acryl ate emulsions have become one of the major research and development fields due to its environmental friendliness, high weather ability, excellent film-processing ability and constructability[1-4].However the obvious disadvantages including poor resistance to heat temperature and little water-resistance restrict its application range[2,5]. In addition, when they were used as architectural coatings and encountered low temperature, they would become fragilely. Thus, to avoid these disadvantages, attempts have been made by some researchers to synthesize a series of novel latexes using sorts of functional monomers to modify[5-8].

Among numerous functional monomers, fluorine-containing monomers have attracted increasing attention of many investigators[7,9,10], because the acryl ate copolymer films modified by them not noly keep the original properties of polyacrylate, such as good adhesion to matrics, but also have their unique and excellent properties, which include high thermal, chemical, aging and weather resistance. Furthermore, they have low levels in dielectric constants, refractive index, surface energy and flammbility, execellent inertness to solvents, hydrocarbons, acids, alkalis and moisture adsorption as well as intriguing oil and water repellency due to the low polarizability and the strong electro-negativity of the fluorine atom[11-12]. Therefore, they have been used progressively in a wide-range of applications[13-15]. However, the price of fluorinated monomers is rather which leads to a limitation of large-scale use for fluorinated polyacrylate(PFA) products.

To control the amount of DFMA, we used a new polymerization technology based on seed emulsion polymerization which was called core-shell emulsion polymerization. We arranged them in the shell part which was coated on of the core part. Herein, the fluorinated polyacrylate emulsion with core-shell structure was prepared via pre-emulsified semi-continuous seeded polymerization using SDS and OP-10 as compound emulsifier, methyl methylacrylate (MMA) and butyl acrylate (BA) as main monomers and dodecafluoroheptyl methacrylate (DFMA) as functional monomers. The effects of polymerization conditions on the conversion and poly-merization stability were studied, meanwhile, FT-IR, SEM, TEM and PSDA (particulate size description analyser) were used to characterize the structure and the product. The results showed that DMFA effectively invovled in the emulsion copolymerization, in addition, uniform particle size and a clear core-shell structure were attained.

\section{EXPERIMENTAL}

\section{A. Material}

Methyl methacrylate (MMA, 99+\%), polyoxyethylated alkyphenol (OP-10, 99+\%), sodium dodecysulfate (SDS, 99+\%) were purchased from Shanghai Lingfeng Chemical Reagent Co. Ltd., China. Butyl acrylate (BA, 99+\%) and Dodecafluoroheptyl methacrylate (Actyflon-G04, 96+\%) were obtained from Sinopharm Chemical Reagent Co. Ltd., China and XEOGIA Fluorine-Silicon Chemical Co. Ltd., China 
respectively. Ammonium persulphate (APS, 99+\%) was purchased from Chinasun Specailty Products Co. Ltd., China. Ammonia Solution (25\%) and filtrating base paper were from Hangzhou Liren Pharmaceutical Co. Ltd., China and a certain Hangzhou Special Paper Co. Ltd., China.

1) Synthetic technology roadmap: Synthetic technology roadmap is shown in Figure 1.

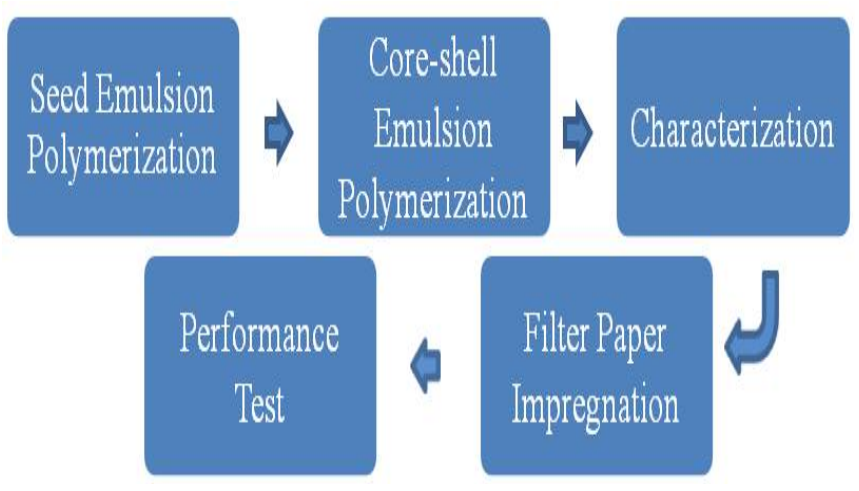

FIGURE I. SYNTHETIC TECHNOLOGY ROADMAP.

\section{2) Core polymerization:}

The reaction was conducted in a $250 \mathrm{~mL}$ four-neck flask equipped with a reflux condenser, a mechanical stirrer, a thermometer, and a nitrogen gas inlet. Core part pre-emulsification: OP-10, SDS and $\mathrm{H} 2 \mathrm{O}$ were added to the reactor as follows. When all the emulsifier solute and reached $40 \mathrm{oC}$, the mixtures added with MMA and BA were pre-emulsified with a high stirring speed under nitrogen atmosphere for about 20 mins.

Seed emulsion polymerization: The mixture was heated to $720 \mathrm{C}$ in a water bath and a part of APS solution (1.6g APS dissolved in $40.0 \mathrm{~g}$ water) was added to trigger reaction. When the mixture in the reactor appeared blue fluorescence, the reaction was kept for another 30 mins and then cooled to room temperature.

\section{3) Shell polymerization:}

Shell part pre-emulsification: OP-10, SDS and H2O were added to a four-neck jacketed glass reactor as above. After the emulsifier stirred and dissolved, certain amount of MMA and DFMA were added.

Core-shell emulsion polymerization: All the shell emulsion were replaced by core emulsion, then the shell emulsion and APS solution were added using the monomer-starved method at $72 \mathrm{oC}$ in about $2 \mathrm{~h}$. When the mixture and APS solution was added completely, the reaction was kept for another $1 \mathrm{~h}$. At last, ammonia solution was dropped into the synthesized emulsion to adjust the $\mathrm{pH}$ value to the range of 6.0-7.0.

\section{4) Impregnation of industrial filter paper:}

Filtrating base paper was impregnated into the above dilute fluorinated acrylate emulsion. The glue amount was controlled at $20 \pm 2 \%$ by press out the redundant impregnation agent from the bottom of filter paper. After dried by electricity heat drum wind drying oven at $110 \mathrm{oC}$, the products were obtained.

5) Water resistance and paper stiffness test: Water resistance test referred to JIS P8122-2004. Paper stiffness test referred to GB/T 22364-2008.

\section{6) Characterization and measurements:}

Conversion rate and gel fraction were tested by weighing method. The emulsion particle size and its distribution were determined by a granularity measurement instrument (Zetasizer Nano ZEN3600) from Malvern Co. (England). Scanning electrons microscope (SEM, Hitachi S-4700II, Japan) was used to investigate the surface morphology of emulsion particles.

\section{B. Results and Discussion}

\section{1) Effect of emulsifier:}

The effect of $\mathrm{m}$ (SDS)/m (OP-10) ratio on the steady-time of the pre-emulsion was shown in Figure 2. As could be seen in the figure, with the increase in the $\mathrm{m}(\mathrm{SDS}) / \mathrm{m}(\mathrm{OP}-10)$ ratio, the emulsion stability increased firstly and then declined. When SDS mass-fraction reached $33.3 \%$, in other words $\mathrm{m}$ (SDS) $/ \mathrm{m}$ (OP-10) ratio equaled 1/2, the steady time stayed the longest. This is because when using the nonionic emulsifier OP-10 and ionic emulsifier SDS as compound emulsifier, they were adsorbed on the surface of latex particles alternatively and SDS molecules were inserted into OP-10 molecules. This resulted that the static tension of the latex particle surface was greatly reduced and the adsorption force with latex particles increased. Simultaneously the thick hydro-layer formed on the surface by OP-10 guarantee the stability of polymer emulsion with the help of synergistic effect [16]. Above all, when SDS/OP-10 equaled $1 / 2$, the combination between OP-10 and SDS seemed best and thus led to a best emulsifying effect.

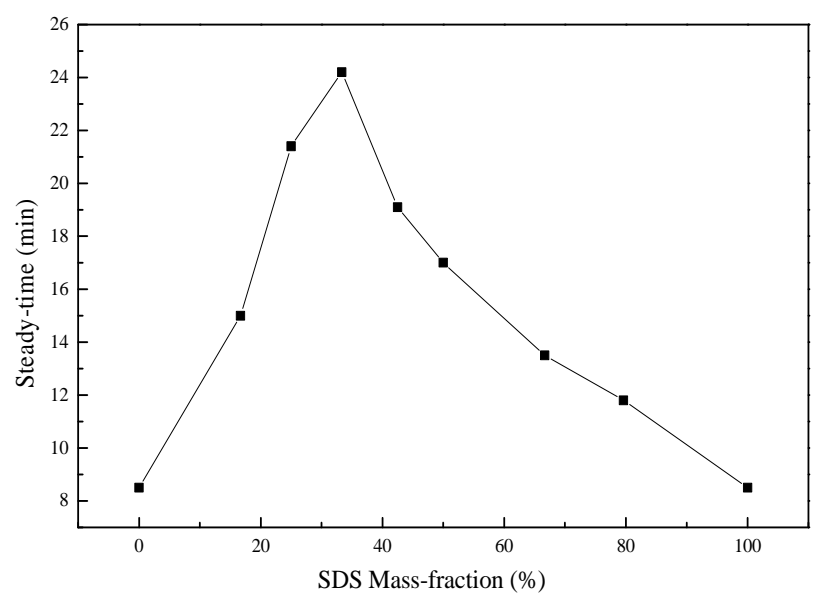

FIGURE II. EFFECT OF M (SDS)/M (OP-10) RATIO ON THE STEADY-TIME OF THE PRE-EMULSION. 


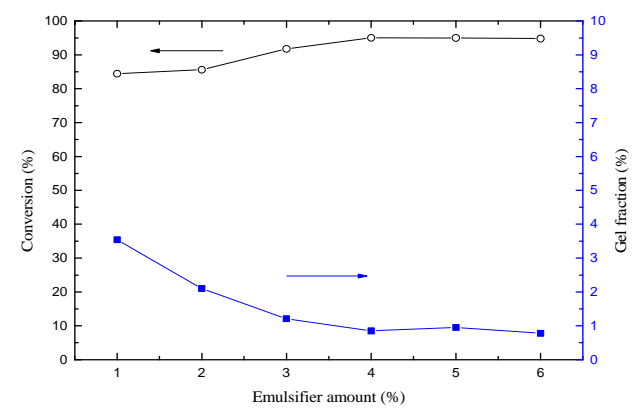

FIGURE III. EFFECT OF EMULSIFIER AMOUNT ON THE CONVERSION AND GEL FRACTION.

As shown in Figure 3 was the effect of emulsifier amount on the conversion and gel fraction. As we see, with the increase of emulsifier dosage, the final conversion of polymer emulsion increased gradually and when the emulsifier dosage arrived at $4.0 \%$, it became balanced. However, the gel fraction was trending downward and varied little after $3.0 \%$. This is partly because the total gel fraction was only a little that the collection of the gel had great chance to bring error. On the other hand, this precisely illustrated when about 3.0\%, the emulsifier had met the requirement of the system stability and the increase of particle size. Overall considered, the compound emulsifier amount was controlled at 3.5(wt) \%-4.0(wt) \% of the monomer amount.

\section{2) Effect of initiator amount:}

Figure 4 showed the effect of APS initiator amount on the final conversion and polymerization stability. As shown here, with the increase of dosage of initiator, both conversion and gel fraction were on the rise as a whole. Gel fraction maintained upward trend, while conversion rate was first increased and then decreased a little.

When the initiator amount arose from $0.15 \%$ to $0.62 \%$, the concentration of free radicals increased in the water phase, so the ones adsorbed onto the particle surface increased. The increasing free radicals ultimately resulted in the progressive reaction and connection between monomers and particles. With the further reaction, the conversion rate improved much higher. However when the dosage of initiator continued to be arise, the polymerization speed was accelerated and thus resulted that the Brownian motion[17] of latex particles was more active which could enlarge the collision rate. But the more active system brought lower polymerization stability, a little lower conversion rate and higher gel fraction.

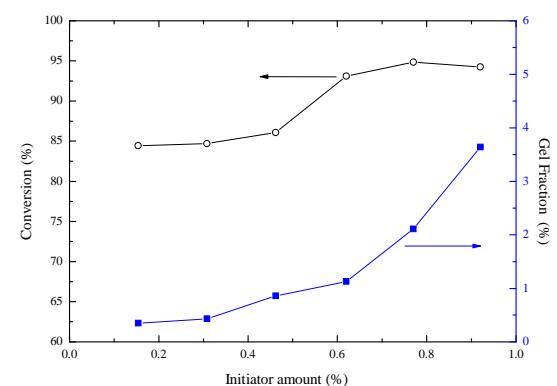

FIGURE IV. EFFECT OF INITIATOR AMOUNT ON THE CONVERSION AND GEL FRACTION.
3) SEM Analysis

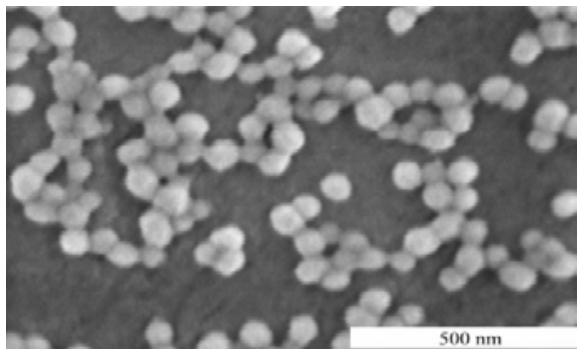

FIGURE V. SEM PHOTOGRAPHS OF FLUORINE-CONTAINING ACRYLATE LATEX PARTICLES.

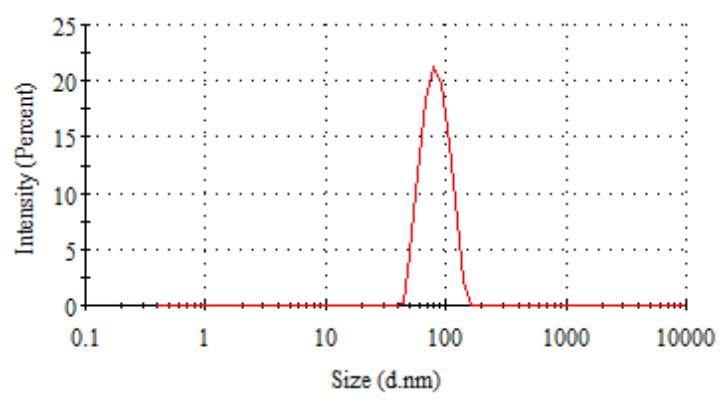

FIGURE VI. PARTICLE SIZE DISTRIBUTION OF FLUORINE-CONTAINING ACRYLATE LATEX PARTICLES.

Figure 5 and Figure 6 were SEM photographs and particle size distribution of fluorine-containing acrylate latex particles. As Figure 5 showed, the latex particle which had uniformly size was mono-dispersed and nearly spherical. As seen in Figure 6, the particle size was about $84 \mathrm{~nm}$, dispersity index PdI was $0.027(<0.06)$ and they also confirmed the above result made from SEM figure. The core-shell polymerization was employed with Semi-continuous Hunger State Changing Mode, the low concentration monomer of core layer on the surface were soon reacted to keep the latex particles' uniformity -- that is why the uniform particles were obtained.

\section{CONCLUSION}

A type of fluorine containing acrylate emulsion with core-shell structure were successfully synthesized using a mixture emulsifier system via an emulsion polymerization approach. While the dosage of the mixture emulsifier, the initiator APS and the monomer DFMA were employed as $4 \%$, $0.6 \%$ and $6 \%$ (on basis of the total monomer weight), $\mathrm{m}(\mathrm{SDS}) / \mathrm{m}(\mathrm{OP}-10)$ equaled $1 / 2$, the latex particles with distinct core-shell structure prepared could be acquired with narrower size distribution, smaller particle size and stronger water resistance.

\section{ACKNOWLEDGEMENT}

This work was supported by the National Natural Science Fund of China (No. 21173193, No. 21301154) and the Commonweal Project from Science Technology Department of Zhejiang Province (No. 2014C33038).

\section{REFERENCES}

[1] A. Bal, G. Guclu, T.B. Iyim, S. Ozgumus, Effects of Nanoparticles on Film Properties of Waterborne Acrylic Emulsions, Polym.-Plast. 
Technol. Eng. 50 (2011) 990-995.

[2] X. Qu, N. Wang, P.A. Lovell, Preparation and Characterization of the Latexes with Different Particle Sizes by Semibatch Emulsion Polymerization and the Influence on Properties of Waterborne Pressure-Sensitive Adhesives, J. Appl. Polym. Sci. 112 (2009) 3030-3040.

[3] K. Zhang, H. Shen, X. Zhang, R. Lan, H. Chen, Preparation and Properties of a Waterborne Contact Adhesive Based on Polychloroprene Latex and Styrene-Acrylate Emulsion Blend, J. Adhes. Sci. Technol. 23 (2009) 163-175.

[4] D. Wu, F. Qiu, H. Xu, J. Zhang, D. Yang, Preparation, Characterization, and Properties of Environmentally Friendly Waterborne Poly(urethane acrylate)/Silica Hybrids, J. Appl. Polym. Sci. 119 (2011) 1683-1695.

[5] J. Xu, H. Hu, Preparation and Characterization of Styrene Acrylate Emulsion Surface Sizing Agent Modified with Rosin, J. Appl. Polym. Sci. 123 (2012) 611-616.

[6] J.Y. Seo, M. Han, Multi-functional hybrid coatings containing silica nanoparticles and anti-corrosive acrylate monomer for scratch and corrosion resistance, Nanotechnology. 22 (2011) 025601.

[7] C. Ai, Y. Ke, Y. Yi, J. Guan, F. Pan, Preparation and Properties of Poly(fluorated-acrylate)/Montmorillonite Composite Emulsion, Integr. Ferroelectr. 136 (2012) 156-168.

[8] H. Li, F. Lei, P. Li, W. Duan, J. Zhou, X. Tan, Selective Adsorption of Berberine Hydrochloride Using Molecularly Imprinted Polymers with Modified Rosin as Cross-linker, Asian J. Chem. 25 (2013) 7421-7426.

[9] L. Zhang, L. Zang, H. Zhang, J. Guo, Synthesis of fluorine-containing latexes with core-shell structure by UV-initiated microemulsion polymerization, Iran. Polym. J. 22 (2013) 93-100.

[10] W. Xu, Q. An, L. Hao, L. Huang, Synthesis, film morphology, and performance of cationic fluorinated polyacrylate emulsion with core-shell structure, J. Appl. Polym. Sci. 125 (2012) 2376-2383.

[11] J. Wang, X.-R. Zeng, H.-Q. Li, Preparation and characterization of soap-free fluorine-containing acrylate latex, J. Coat. Technol. Res. 7 (2010) 469-476.

[12] L. Junyan, H. Ling, Z. Yuansuo, Synthesis and Property Investigation of Three Core-Shell Fluoroacrylate Copolymer Latexes, J. Appl. Polym. Sci. 112 (2009) 1615-1621.

[13] X. Huang, X. Wen, J. Cheng, Z. Yang, Sticky superhydrophobic filter paper developed by dip-coating of fluorinated waterborne epoxy emulsion, Appl. Surf. Sci. 258 (2012) 8739-8746.

[14] T. Kubota, Recent Development of Fluorine-containing Transparent Polymers, J. Synth. Org. Chem. Jpn. 71 (2013) 196-206.

[15] Z.-Y. Wang, J.-C. Ho, W.-J. Shu, Studies of fluorine-containing bismaleimide resins part I: Synthesis and characteristics of model compounds, J. Appl. Polym. Sci. 123 (2012) 2977-2984.

[16] T. Cao, Q. Liu, J. Hu, Synthesis Principle, Property and Application of Polymer Emulsion, second ed., Chemical Industry Press, Beijing, 2007, pp. 172-256.

[17] T. Poggio, V. Kapeliouchko, V. Arcella, E. Marchese, Multimodal fluoropolymer dispersions, Prog. Org. Coat. 48 (2003) 310-315. 